\title{
Large-Scale Open Pool Experimental Data and Analysis for Fire Model Validation and Development
}

\author{
THOMAS BLANCHAT and VICTOR FIGUEROA \\ Fire and Aerosol Sciences Department \\ Sandia National Laboratories \\ P.O. Box 5800 \\ Albuquerque, NM 87185-1135
}

\begin{abstract}
Four large-scale open pool fire experiments were performed with well-characterized boundary and initial conditions. Results presented include a general description of test observations, wind measurements, fire plume topology, fuel recession and heat release rates, incident heat flux to the pool, surrounding terrain, and calorimeters. All initial and boundary condition data required as necessary inputs to computation models are also presented. The large physical scale, the experimental design, the use of independent measurement techniques, and the attention to data quality provide a unique dataset to support numerical fire model validation.
\end{abstract}

KEYWORDS: pool fires, calorimetry, heat flux, heat release rate, CFD

\section{INTRODUCTION}

Sandia National Laboratories (SNL) and the Defense Threat Reduction Agency (DTRA) established a Fuel Fire Technology Base Program with the goal to develop validated numerical tools that model dominant physical phenomena necessary to predict the thermal hazard posed by fuel fires from aircraft or ground transportation accidents. The continued development of the technology to provide validated fire physics models will eventually lead to an ability to predict the threat posed by fires, the response of objects in fires, and confidence in those predictions. The focus of the Well-Characterized Open Pool fire series described in this paper and other previous SNL fire experiments [1-7] is to provide archival experimental data of sufficient quality for CFD fire code development and validation purposes. Four fire experiments were conducted with two different wind conditions. Test 1 and Test 2 were conducted with a large calorimeter external to the pool and Test 3 and Test 4 were conducted without the calorimeter. The experiments measured the burning rate of liquid fuel in an open pool and the resultant heat flux to various objects and the surrounding environment with well-characterized boundary and initial conditions. Extensive video was used to determine flame height and drag.

\section{EXPERIMENTAL SETUP}

The tests were performed in a $7.92 \mathrm{~m}$ diameter, outdoor-pool fire test facility located at Sandia National Laboratories Lurance Canyon Burn Site. The circular fuel pool (mild steel, $1.3 \mathrm{~cm}$ wall thickness, $\sim 92 \mathrm{~cm}$ deep) was sunken, flush with the surrounding terrain, as shown in Figure 1. All tests utilized JP8 fuel. Four calorimeters and 49 Sandia Heat Flux Gauges (HFGs) located both inside and outside the fire were used to measure object, pool, and surrounding terrain incident heat flux. All calorimeters were positioned such that prevailing winds (typically from $265^{\circ}$ ) were normal to the long axis of the large calorimeter. The large calorimeter was positioned at the lee side (east edge) of the open pool. This position was chosen in part based on results from a previous test series where the presence of a cylinder at the lee side of a wind-swept flame zone had a pronounced effect on the fire as compared to fires without engulfed objects. Four towers ( $\sim 18 \mathrm{~m}$ separation between each tower) located about $38 \mathrm{~m}$ upwind from the pool centerline were used to collect wind data. Wind sensors were placed at 2, 5, and 10 meters above the ground on each tower.

\section{Fuel Surface and Terrain Heat Flux Measurements}

Hemispherical heat flux gauges (HFG), designed by SNL, were used to measure the spatial and temporal distribution of the heat flux near the fuel surface and the surrounding terrain. The HFG is primarily a thin metal plate with one side facing the fire environment and the other side insulated. The plate temperature is 
measured using a thermocouple (TC) attached to the insulated face of the metal plate. Experimental analyses have determined that applying a simple thermal response model to the SNL HFG data yields incident heat fluxes to within about $5 \%$ of independently measured values, provided the input flux is steady. However, as discussed later, the uncertainty in the measurement can be large. The response time of the HFG has been experimentally determined to be on the order of about 60 seconds. Details of the construction and the performance of the gauge, the thermal response model used to reduce the HFG data, and the uncertainty analysis of the thermal response model are described in the SNL HFG report [8]. As illustrated in Figure 1, forty-nine single-sided, upward facing, HFGs were used. The gauge sensing face was located approximately $15 \mathrm{~cm}$ above the terrain surface and approximately $18 \mathrm{~cm}$ above the liquid fuel surface. Figure 1 also shows the large calorimeter used in Tests 1 and 2 on the east side of the pool.
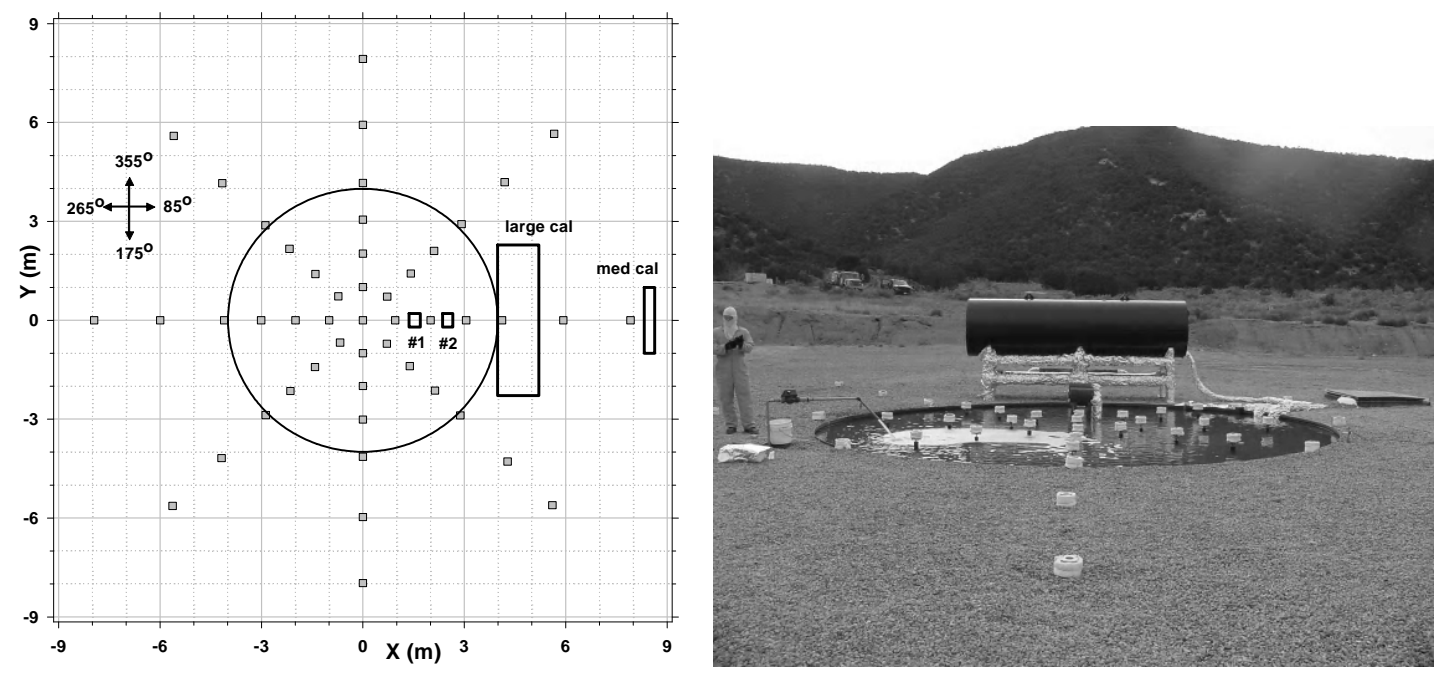

Fig. 1. Pool layout - location of HFGs and calorimeters.

\section{Small Calorimeters}

Two Sandia-designed calorimeters were placed within the pool approximately $16 \mathrm{~cm}$ (from the calorimeter bottom) above the ground plane surface. Each calorimeter is a stainless steel (SS) cylinder $30.5 \mathrm{~cm}$ in outside diameter, $40.6 \mathrm{~cm}$ long, with $3 \mathrm{~mm}$ wall thickness and $6.4 \mathrm{~cm}$ thick SS end caps. Six TCs were spot welded to the inner surface, four at the cylinder centerline (every 90 degrees) and one in the center of each end cap. The inside is packed with multiple layers of $2.54 \mathrm{~cm}$ thick ceramic fiber (Kaowool, $128 \mathrm{~kg} / \mathrm{m}^{3}$ ) insulation. All external surfaces were painted black using Pyromark 2500 to provide a known emissivity (0.86 \pm 0.09$)$ [9]. Small calorimeter \#1 was located $1.52 \mathrm{~m}$ from pool centerline and $15 \mathrm{~cm}$ above the ground plane. Small calorimeter \#2 was located $2.51 \mathrm{~m}$ from pool centerline and $18 \mathrm{~cm}$ above the ground plane. Both calorimeters were placed to the lee (east) side of the pool, as shown in Fig. 1.

\section{Large Calorimeter}

A large calorimeter, similar in size and shape to a truck sized waste transportation container, simulating a bluff body obstruction, was placed on the lee (east) side of the pool surface, $3.96 \mathrm{~m}$ from pool centerline and with the bottom $0.91 \mathrm{~m}$ above the ground plane for Tests $1 \& 2$. The carbon-steel calorimeter $(1.22 \mathrm{~m}$ diameter, $4.57 \mathrm{~m}$ long, $2.54 \mathrm{~cm}$ wall thickness) has end caps (2.54 cm thick) welded to one end and bolted to the other end. The calorimeter weighs $3860 \mathrm{~kg}$. Sixty-three (63) TCs were attached to inside surfaces at four section lines. Figure 2 shows the locations of the thermocouples. All of the TCs (with the exception of the 200 series located at section 2) are covered with a pad of ceramic fiber insulation $(7.6 \mathrm{~cm}$ thick, $7.6 \mathrm{~cm}$ diameter). For the 200 series, two TCs are used; one TC is tacked to the inside surface with the second TC directly opposite the first, separated by a pad of ceramic fiber insulation $(2.54 \mathrm{~cm}$ thick, $7.6 \mathrm{~cm}$ diameter). The $2^{\text {nd }}$ TC provides an additional boundary condition measurement for determining heat flux using inverse heat conduction methodologies. All external surfaces were painted black using Pyromark 2500. The calorimeter alignment was with Section 4 on the north side of the pool. 


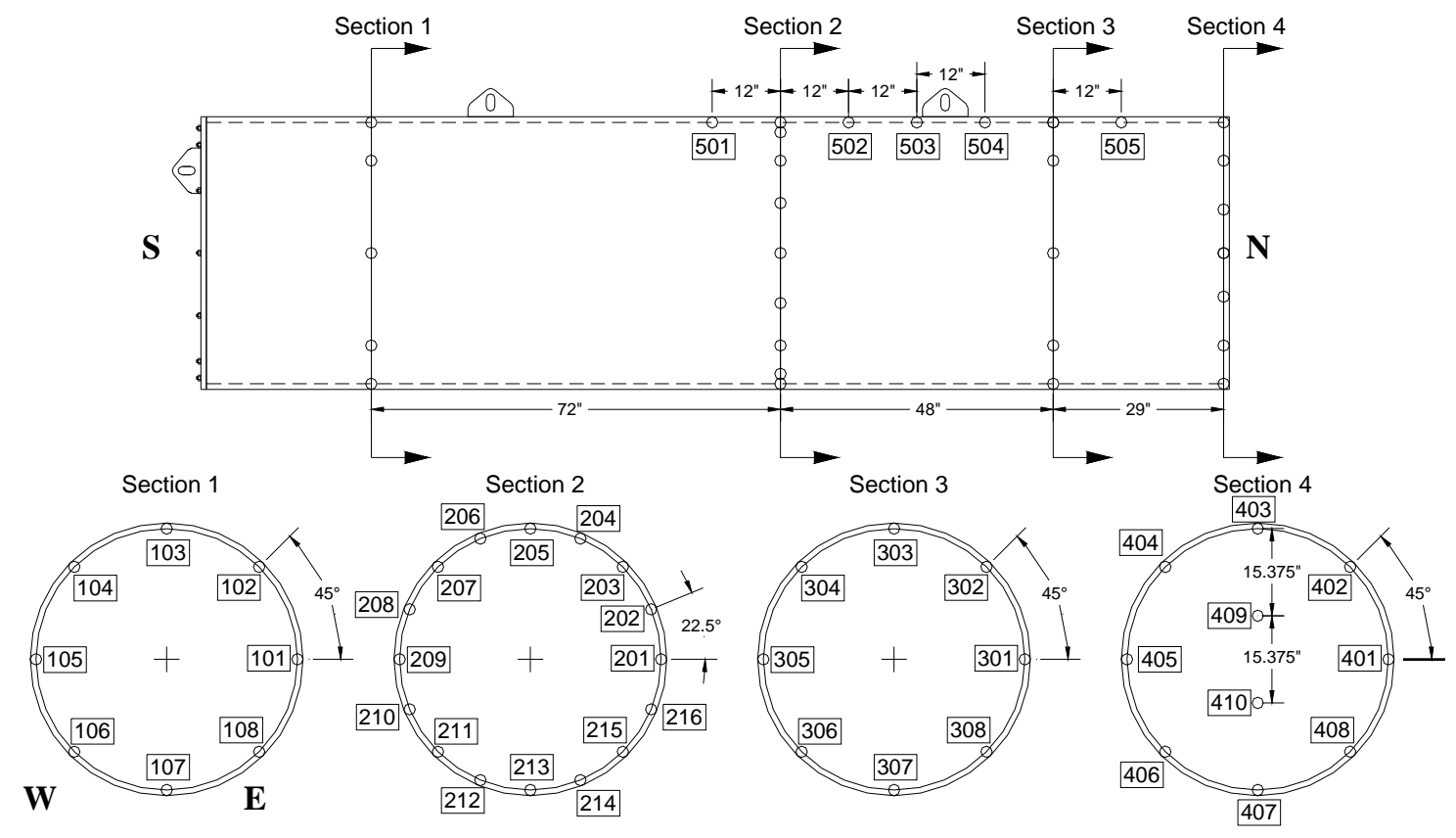

Fig. 2. Large calorimeter thermocouple locations.

\section{Medium Calorimeter}

The medium calorimeter was constructed of $3.2 \mathrm{~mm}$ thick 304 stainless steel. It has an outer diameter of $30.5 \mathrm{~cm}$ and is $2 \mathrm{~m}$ long. It was located $8.5 \mathrm{~m}$ on the lee side from pool centerline and with the bottom 0.3 $\mathrm{m}$ above the ground plane. There are 8 circumferential measurement stations at the calorimeter midplane. Measurement station 1 faces east with the rest sequentially numbered in a counterclockwise direction when facing north. Each station consisted of a square coupon (3.2 mm thick) fitted with an intrinsic TC on the internal surface. A layer of $2.54 \mathrm{~cm}$ thick ceramic fiber insulation covered the TC. Another TC (attached to $1 \mathrm{~mm}$ stainless shim stock) was directly opposite and separated from the coupon TC by a layer of $2.54 \mathrm{~cm}$ thick ceramic fiber insulation. The rest of the volume was filled with ceramic fiber insulation. The calorimeter body (center section) has been painted with Pyromark 2500.

\section{Wind Speed and Direction, Fuel Recession, and Video}

Wind speed and direction were measured sufficiently far from the boundary of the pool to reduce the influence of air entrained by the fire plume from the surrounding environment and mitigate the potential effects of radiant heat from the fire. Twelve ultrasonic anemometers (Young 81000) were used to obtain three dimensional wind velocities.

The rate of liquid fuel vaporization is among the most important variables to be predicted in the simulation of fire environments. The fuel vaporization, or burn rate, determines the amount of fuel available for combustion within the flame zone, the heat release rate (HRR), and also defines the duration of the fire. Data acquired from earlier test series in this program indicate that the rate of fuel vaporization becomes constant with time shortly after ignition, but varies over the surface of the fuel pool. This spatial variation may be induced by non-uniform fuel surface heat flux and thermal transport within the fuel.

Burning (mass loss) rate was determined by measuring the transient liquid pool head pressure (Rosemount Model 3051 differential pressure gauge, 0-64 cm water column WC). Note that it was necessary to route a low-pressure DP line to a point above the pan surface to reduce the cyclic pressure pulse due to the flame "puffing". The fuel temperature was measured using a TC "rake". The fuel rake can also be used to determine fuel recession, based on a time-of-arrival analysis, a technique used and proven many times in previous experiments. Fuel temperature affects the burn rate and thus becomes an important parameter to 
measure. The fuel pool used one vertically mounted TC rake containing $30 \mathrm{TCs}(1.3 \mathrm{~cm}$ spacing between TCs). At the start of the fire, the top 2 or 3 fuel rake TCs were above the fuel surface and 12 TCs were in the $\sim 15.2 \mathrm{~cm}$ fuel layer of JP-8. Except where indicated, all temperature measurements utilized $1.6 \mathrm{~mm}$ diameter, Inconel sheathed, ultra-pure MgO insulated type-K TCs, with ungrounded junctions. The response time of these TCs in a fire is expected to be on the order of a few seconds.

Five video cameras placed North, Northeast, East, Southeast and South around the pool were used to study the fire plume topology. The cameras were positioned between $61 \mathrm{~m}$ and $91 \mathrm{~m}$ from pool centerline, yielding a vertical field of view from the pool surface to an elevation of approximately $55 \mathrm{~m}$ above the pool surface.

\section{Data Acquisition and Uncertainty}

Data acquisition (DAQ) was PC-based using 16-bit National Instruments (NI) data acquisition hardware. Thermocouple and heat-flux data were sampled simultaneously for all channels, typically at $1000 \mathrm{~Hz}$ with an average value recorded at a rate of at least one sample per second. Channel integrity was evaluated prior to each experiment with a calibrated voltage source. All TC channels had a pretest uncertainty to within $\pm 1.7 \mathrm{~K}$. However, for fires of this magnitude, thermocouple shunting can introduce considerable temperature measurement error [10]. The uncertainty of the entire DAQ (and associated instrumentation) was estimated by assessing the individual uncertainties of each component, then combining them using the root-sum-square (RSS) method [11].

\section{EXPERIMENTAL RESULTS}

\section{Test Conditions}

Results presented here include a general description of test observations, wind measurements, fire plume topology, average fuel recession and heat release rates, and incident heat flux to the pool and to the calorimeters. Average wind conditions are given and were used to identify quasi-steady periods. Heat flux contour plots were generated for quasi-steady periods for the pool and for the large calorimeter. The ambient air temperature and fuel temperature ranged between $17-30^{\circ} \mathrm{C}$ and $11-30^{\circ} \mathrm{C}$, respectively. The ambient air pressure was $\sim 0.08 \mathrm{MPa}$ for all tests.

All tests were performed using approximately 2207 gallons of JP8 aviation fuel floated on $76 \mathrm{~cm}$ of water, resulting in a liquid pool surface very near the surrounding terrain surface at the start of the test. As the fire burns, the fuel level drops with time until at the end of the test the fuel surface is $\sim 15.2 \mathrm{~cm}$ below the ground plane. Since the regression rate is measured, the location of the fuel surface with respect to the ground plane is known at all times. In small fires in quiescent laboratory conditions, fuel pool lip effects are quite important because they induce a transition to turbulence. In large outdoor fires the effect is expected to be much less important.

\section{Wind Measurements and Observations}

Table 1 summarizes wind speeds and wind directions at the $10 \mathrm{~m}$ height (average of the four ultrasonic anemometers over the test duration from $\mathrm{t}=0-2700 \mathrm{~s}$ ) in the test series. Wind conditions were nearly calm in Test 1 . The fire plume was approximately normal to the ground during most of the test. Changes in wind direction tipped the plume slightly in various directions and caused the east edge of the plume to lean towards or away from the large calorimeter from time to time. Soot coverage was limited to the area near the pool due to the relatively calm wind conditions. 
Table 1. Average Wind Conditions at 10-m Height.

\begin{tabular}{|c|c|c|c|c|}
\hline \multirow{2}{*}{ Test } & \multicolumn{2}{|c|}{ Wind Speeds (m/s) } & \multicolumn{2}{c|}{ Wind Direction (degrees) } \\
\cline { 2 - 5 } & Mean & Std. Deviation & Mean & Std. Deviation \\
\hline 1 & 0.85 & \pm 0.27 & 178.5 & \pm 76.6 \\
\hline 2 & 5.76 & \pm 1.52 & 246.9 & \pm 15.0 \\
\hline 3 & 1.66 & \pm 0.34 & 102.6 & \pm 36.8 \\
\hline 4 & 3.83 & \pm 0.97 & 237.0 & \pm 12.7 \\
\hline
\end{tabular}

Test 2 had the highest wind speeds. The fire plume tilted significantly from the ground normal $\left(>45^{\circ}\right)$ in the wind direction during most of the test. The fire plume covered the large calorimeter and the medium calorimeter during most of the test. Posttest observation showed a significant soot outline on the pool east side that indicated the extent of the plume contact with the terrain. The soot footprint extended approximately half a diameter from the edge of the pool in the direction toward the medium calorimeter. Test 3 was a low wind speed test. As in Test 1 , wind direction varied significantly and the fire plume remained close to the vertical position with respect to the ground plane during most of the test. The soot outline on the ground indicated the plume direction was tilted slightly toward the northwest side of the pool (wind direction was predominantly from the southeast). Test 4 was a high wind speed test. The fire plume tilted significantly from the ground normal during most of the test and swayed from northeast to the east. The soot outline on the ground also indicated the plume direction was toward the east side of the pool.

\section{Flame Topology}

Fire plume structure sizes (the maximum height and length of the visible flame) were determined from triangulation with two cameras and utilizing surveyed distances between cameras, two fiducials in the camera field of view, and various points of interest around the test site. Tests 1 and 3 yielded $\sim 18-21 \mathrm{~m}$ plume heights and lengths (maximum distance from the pool center over time), as expected with a near vertical plume. Both maximum height and distance from center of the pool were fairly constant due to the relatively calm wind conditions. The maximum heights in Tests 2 and 4 varied in the range 9-15 m depending on the wind fluctuation and intensity. The plume lengths in Test 2 were 12-24 $\mathrm{m}$ whereas in Test 4 the plume lengths were 9-15 $\mathrm{m}$.

\section{Fuel Recession Rates and Heat Release Rates (HRR)}

Fuel recession rates for the liquid fuel in the pool were calculated from the temperature history of the thermocouple array. The fuel recession rate is calculated by dividing the TC spacing by the time it took sequential TCs to reach the fuel boiling temperature $\left(150^{\circ} \mathrm{C}\right)$. Fuel recession rates for the pool were also obtained by measuring the liquid pool head differential pressure (DP). Table 2 shows fuel recession rates obtained from thermocouple array and from DP measurements (DP data was unreliable in Test 1).

Table 2. Pool Fuel Recession, Mass Loss Rate, and HRR.

\begin{tabular}{|l|c|c|c|c|c|}
\hline & Uncertainty & Test 1 & Test 2 & Test 3 & Test 4 \\
\hline Recession DP (mm/min) & $0.6 \%$ & - & 5.17 & 4.53 & 5.02 \\
\hline $\begin{array}{l}\text { Recession TC array } \\
(\mathrm{mm} / \mathrm{min})\end{array}$ & $4.3 \%$ & 4.32 & 5.28 & 4.64 & 5.03 \\
\hline Mass Loss Flux $\left(\mathrm{kg} / \mathrm{m}^{2} \mathrm{~s}\right)$ & $4.1 \%$ & 0.058 & 0.071 & 0.062 & 0.068 \\
\hline Heat Release Rate $(\mathrm{MW})$ & $9.1 \%$ & 124 & 151 & 133 & 144 \\
\hline $\begin{array}{l}\text { Pool Surface Heat Flux } \\
\left(\mathrm{kW} / \mathrm{m}^{2}\right)\end{array}$ & $\pm 25-42 \%$ & $78 \pm 9$ & $97 \pm 5$ & $85 \pm 7$ & $94 \pm 6$ \\
\hline Wind Speed $(\mathrm{m} / \mathrm{s})$ & 0.85 & 0.85 & 5.76 & 1.66 & 3.83 \\
\hline
\end{tabular}

Fuel recession rates obtained using fuel temperature measurements and DP measurements are in good agreement. An uncertainty analysis for the fuel recession using TC and DP measurements was performed using the method described by Coleman and Steele [11]. The uncertainty for fuel regression is strongly dependent on the systematic uncertainty. Parameters contributing to the systematic uncertainty are primarily based on manufacturer's specifications. Contributions to the error for fuel recession by the TC 
rake method include the TC spacing error (4\%) and the time measurement uncertainty $(0.3 \%)$. Contributions to the error for fuel recession by the DP method include the pressure measurement uncertainty $\left(0.4 \%\right.$ for temperature changes no greater than $\left.28^{\circ} \mathrm{C}\right)$, the time measurement uncertainty $(0.03 \%)$, and the fuel specific gravity measurement uncertainty $(0.5 \%)$, all taken as a percentage of the upper limit of the gauge. The calculated total uncertainty in the fuel regression measurement due to systematic uncertainty is $0.4 \mathrm{~cm} \mathrm{WC}$, or $0.6 \%$ of the maximum value for the gauge.

The fuel recession rates ranged between $4.32 \mathrm{~mm} / \mathrm{min}$ and $5.28 \mathrm{~mm} / \mathrm{min}$. These measurements are consistent with what is typically observed in large pool fires [12]. Mass loss fluxes were calculated by multiplying the fuel recession rate by the fuel density $\left(808 \pm 4 \mathrm{~kg} / \mathrm{m}^{3}\right)$. Table 2 presents this mass flux and the total heat release rate (HRR), with the latter determined by multiplying the mass flux by the JP- 8 fuel heat of combustion $(43.2 \pm 3 \mathrm{MJ} / \mathrm{kg})$ and by the pan area $\left(49.3 \pm 2.0 \mathrm{~m}^{2}\right)$. The data shown in Figure 3 suggest a strong linear correlation between wind speed and mass loss rate. Table 2 also shows the temporally and spatially averaged heat flux (discussed in the following section) to the pool surface as a function of wind speed.

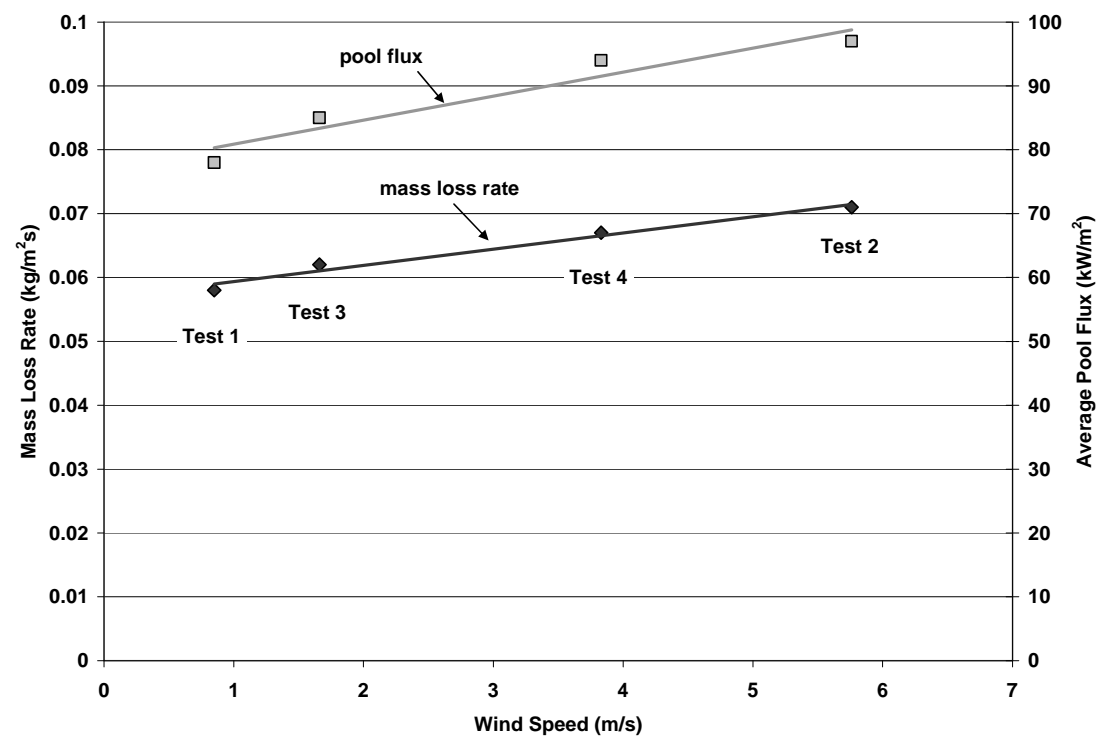

Fig. 3. Mass loss rate and pool flux as a function of wind speed.

\section{Fuel Pool and Surrounding Terrain Heat Fluxes}

HFG data from the fuel surface were post-processed to obtain spatially-resolved estimates of heat transfer to the surface of the pool. The heat balance on the heated surface of an idealized one-dimensional heat flux gage is given in Eq. 1:

$\alpha q_{\text {surf }}(t)=\varepsilon q_{\text {rad }}(t)+q_{\text {steel }}(t)+q_{\text {insul }}(t)+q_{\text {conv }}(t)$

where $q_{\text {sur }}(t)$ is the heat flux incident to the HFG heated plate, $q_{\text {rad }}(t)$ represents the heat re-radiated from the surface of the plate, $q_{\text {steel }}(t)$ is the heat stored in the plate, $q_{\text {insul }}(t)$ is the heat loss to the insulating material (Kaowool), and $q_{\text {conv }}(t)$ is the convective heat loss. The absorptivity $(\alpha)$ and the emissivity $(\varepsilon)$ are assumed equal to 0.86 . The convective heat component of the incident heat flux, $q_{\text {conv }}(t)$, was not considered in the data reduction. Due to the lack of free stream gas velocity and gas temperature measurements, the convection heat transfer was treated as an uncertainty of incident heat flux calculations. The methodology for estimation of the uncertainty estimates for HFG measurements is given in [13]. For typical large pool fires, incident heat flux measurement uncertainty for the thin plate/energy balance method is approximately $\sim 25 \%$ during high wind conditions and $\pm 42 \%$ for low wind conditions. This estimate was obtained using large pool fire experiment data and is only valid for steady-state conditions. 
For rapid transient conditions, such as during the pool ignition, the uncertainty is expected to be higher. Figure 4 shows average incident heat flux to the pool surface determined by spatially-averaging the 25 pool HFG measurements.

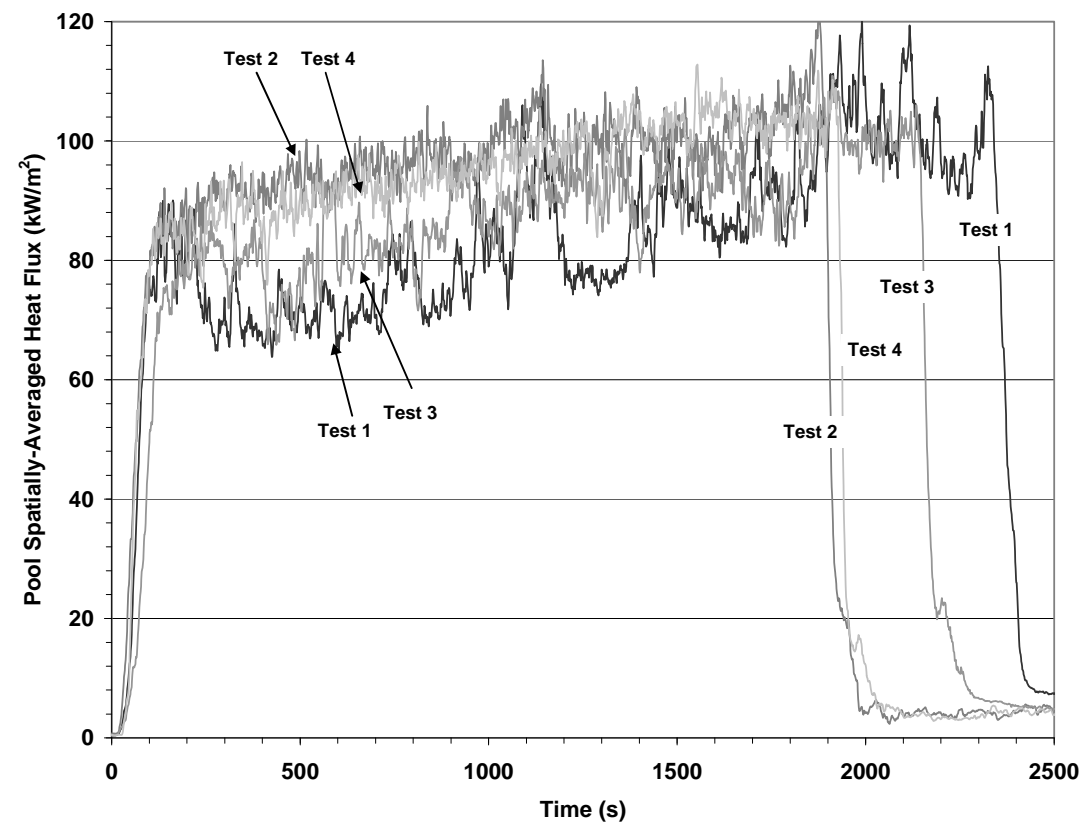

Fig. 4. Spatially-averaged incident heat fluxes obtained from pool HFG measurements.

The average flux across the pool reached steady-state conditions $\sim 100$ seconds after ignition. Burnout occurred sometime after 1900 seconds. The low wind test conditions in Test 1 and 3 produced lower fuel burn rates that prolonged the fire. Figure 4 shows the flux increasing during the tests. This increase in incident heat flux has been observed in previous experiments [14]. The heat fluxes were consistently higher in high wind tests, i.e., Test 2 and Test 4. High wind conditions generally result in higher fuel vaporization rates and thus higher incident heat flux feedback to the pool. Table 2 gives the spatially and temporally averaged flux and standard deviation during the 200-1500 s interval. The average flux varied from a low of $\sim 70 \mathrm{~kW} / \mathrm{m}^{2}$ for Tests 1 and 3 up to $\sim 100 \mathrm{~kW} / \mathrm{m}^{2}$ for Tests 2 and 4 . The standard deviation $(1 \sigma)$ for the spatially-averaged incident heat flux at each time step was in the range of $20-30 \mathrm{~kW} / \mathrm{m}^{2}$ for all tests.

Flux contour maps (Figure 5) for the pool and surrounding terrain were generated at all time steps and also over 1-minute periods of quasi-steady state wind conditions to examine spatial variations. Figure 5 shows that the incident heat fluxes were generally largest inside the pool (note that the average wind direction and speed are also shown in Figure 5). Test 2 (and somewhat less in Test 4) is a notable exception where significantly higher heat fluxes were observed to the surrounding terrain on the leeward side of the pool. In Test 2, maximum incident heat fluxes in the terrain near the medium calorimeter reached $150 \mathrm{~kW} / \mathrm{m}^{2}$. In general, heat fluxes near the edge of the pool were low, especially on the upwind side.

In many calm wind fires, an oxygen starved region exists near the fuel surface in the interior of the fire [4]. This is observed in the contour plot of Test 1 where the winds were relatively calm and the plume was near vertical during most of the test. Regions near the center receive little heat feedback to the pool due to poor combustion, while regions near the edge of the pool receive significantly more heat feedback due to eddies that form near the edges of the plume, enhancing combustion. In general, the extent and location of the oxygen starved region is expected to change with the size of the pool and wind conditions. Previous tests show that, with slightly higher wind speeds $(\sim 1.2 \mathrm{~m} / \mathrm{s})$, the extent of the oxygen starved region is much smaller and pushed slightly upward. 


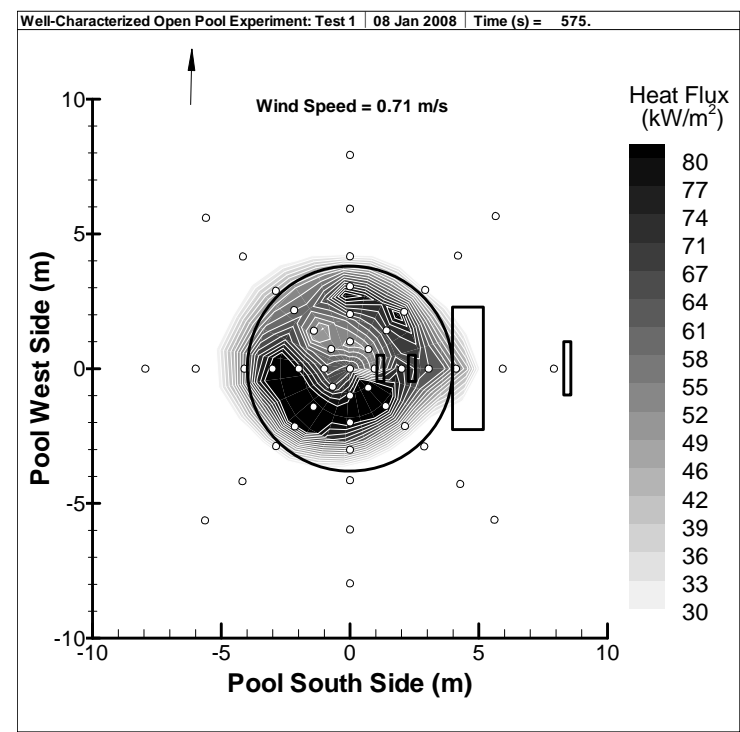

Test 1

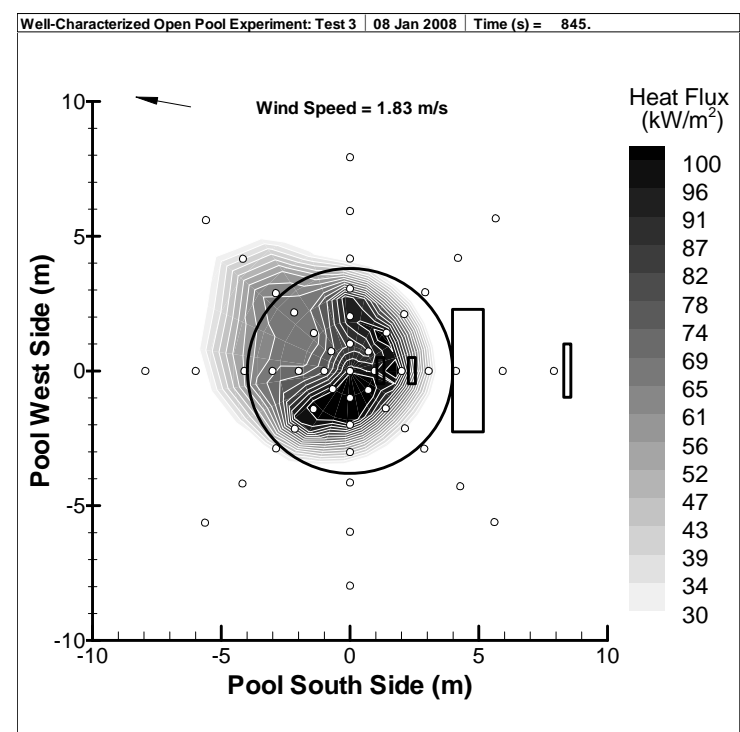

Test 3

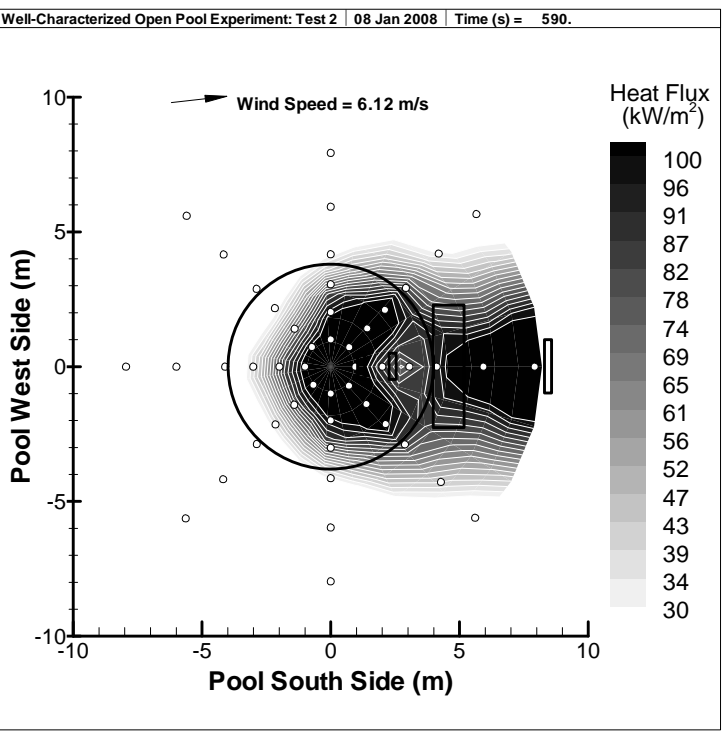

Test 2

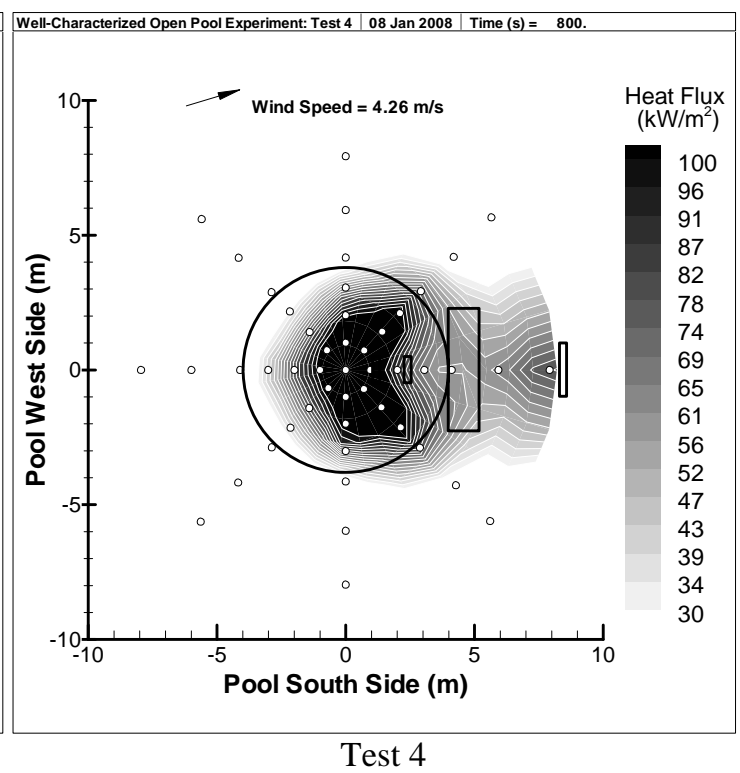

Fig. 5. Spatial variation of the incident heat flux to the pool and terrain.

In Tests 2 and 4, crosswinds tilted the plume away from the ground normal resulting in high incident heat flux regions toward the leeward side of the pool. In general, the reduced heat flux (possibly oxygen starved) region is missing from the center region of contour plots in these figures. In some instantaneous contour plots there are subtle discontinuities; sometimes a U-shape geometry is produced. In crosswinds, rotational structures are formed on the leeward side of the plume, which are different from the upwind side structure. On the upwind side, rotational structures are similar to those found in single column, vertical pool fires.

\section{Small and Medium Calorimeter Heat Flux}

Table 3 presents the heat flux data from both small calorimeters in all tests. Data was averaged over the test durations times listed in the table. A standard deviation on each average is also presented; fairly large standard deviations highlight the highly fluctuating flux seen in these fire environments. Fluxes at some locations are not presented due to TC failures. Peak incident heat fluxes ranged between 125 and 150 $\mathrm{kW} / \mathrm{m}^{2}$. Note that objects in fires are heated very non-uniformly in both space and time. The ratio of the top (facing the flames) to bottom (facing the pool surface) time average fluxes is of the order of 1 to 4 . Thus, 
furnace tests with uniform heating that are used to assess structural response to fire loads should be seen in the light that, more often than not, the time-averaged loads in real fires are spatially non-uniform.

Table 3. Small Calorimeter Average Heat Flux.

\begin{tabular}{|c|c|c|c|c|c|c|c|r|}
\hline & \multicolumn{2}{|c|}{ Test 1 } & \multicolumn{2}{c|}{ Test 2 } & \multicolumn{2}{c|}{ Test 3 } & \multicolumn{2}{c|}{ Test 4 } \\
\hline & $\begin{array}{c}\text { Cal 1 } \\
\text { (west) }\end{array}$ & $\begin{array}{c}\text { Cal 2 } \\
\text { (east) }\end{array}$ & $\begin{array}{c}\text { Cal 1 } \\
\text { (west) }\end{array}$ & $\begin{array}{c}\text { Cal 2 } \\
\text { (east) }\end{array}$ & $\begin{array}{c}\text { Cal 1 } \\
\text { (west) }\end{array}$ & $\begin{array}{c}\text { Cal 2 } \\
\text { (east) }\end{array}$ & $\begin{array}{c}\text { Cal 1 } \\
\text { (west) }\end{array}$ & $\begin{array}{c}\text { Cal 2 } \\
\text { (east) }\end{array}$ \\
\hline $\begin{array}{c}\text { Duration } \\
\text { (s) }\end{array}$ & $\begin{array}{c}200- \\
1500\end{array}$ & $\begin{array}{c}200- \\
1500\end{array}$ & $\begin{array}{c}200- \\
1200\end{array}$ & $200-690$ & $\begin{array}{c}200- \\
1500\end{array}$ & $\begin{array}{c}200- \\
1500\end{array}$ & $\begin{array}{c}200- \\
1500\end{array}$ & $\begin{array}{c}200- \\
1500\end{array}$ \\
\hline Flux & $\begin{array}{r}\text { ave } \pm \text { s.d. } \\
\left(\mathrm{kW} / \mathrm{m}^{2}\right)\end{array}$ & $\begin{array}{r}\text { ave } \pm \text { s.d. } \\
\left(\mathrm{kW} / \mathrm{m}^{2}\right)\end{array}$ & $\begin{array}{c}\text { ave } \pm \text { s.d. } \\
\left(\mathrm{kW} / \mathrm{m}^{2}\right)\end{array}$ & $\begin{array}{r}\text { ave } \pm \mathrm{s} . \mathrm{d} . \\
\left(\mathrm{kW} / \mathrm{m}^{2}\right)\end{array}$ & $\begin{array}{c}\text { ave } \pm \mathrm{s} . \mathrm{d} . \\
\left(\mathrm{kW} / \mathrm{m}^{2}\right)\end{array}$ & $\begin{array}{c}\text { ave } \pm \text { s.d. } \\
\left(\mathrm{kW} / \mathrm{m}^{2}\right)\end{array}$ & $\begin{array}{c}\text { ave } \pm \text { s.d. } \\
\left(\mathrm{kW} / \mathrm{m}^{2}\right)\end{array}$ & $\begin{array}{r}\text { ave } \pm \text { s.d. } \\
\left(\mathrm{kW} / \mathrm{m}^{2}\right)\end{array}$ \\
\hline Top & $125 \pm 25$ & $107 \pm 29$ & - & $155 \pm 12$ & $91 \pm 22$ & - & $158 \pm 16$ & $147 \pm 16$ \\
\hline Bottom & $30 \pm 13$ & $65 \pm 18$ & $92 \pm 16$ & $63 \pm 12$ & $81 \pm 14$ & $62 \pm 12$ & $82 \pm 18$ & $70 \pm 17$ \\
\hline East & $86 \pm 29$ & $107 \pm 25$ & $111 \pm 21$ & $85 \pm 19$ & $100 \pm 13$ & $45 \pm 10$ & $92 \pm 22$ & $79 \pm 26$ \\
\hline West & $56 \pm 20$ & $79 \pm 27$ & $131 \pm 11$ & $124 \pm 12$ & $102 \pm 20$ & $117 \pm 9$ & $130 \pm 13$ & $118 \pm 22$ \\
\hline $\begin{array}{c}\text { North } \\
\text { endplate }\end{array}$ & $72 \pm 26$ & $94 \pm 24$ & $131 \pm 12$ & $114 \pm 19$ & $122 \pm 17$ & $75 \pm 16$ & $134 \pm 22$ & $92 \pm 20$ \\
\hline $\begin{array}{c}\text { South } \\
\text { endplate }\end{array}$ & $73 \pm 33$ & $75 \pm 40$ & - & $86 \pm 10$ & $120 \pm 11$ & $83 \pm 16$ & $131 \pm 20$ & $106 \pm 22$ \\
\hline
\end{tabular}

Table 4 presents the average and standard deviation of the incident heat flux from the medium calorimeter in all tests. Incident heat fluxes to the medium calorimeter were very small for Test 1 and 3 and large for Test 2 and 4. For Test 1 and 3, the fire plume remained close to the ground normal; heat flux to the medium calorimeter was dominated by radiation from the fire plume and was not significant due to the firecalorimeter distance. Strong crosswinds in Tests 2 and 4 tilted the fire plume in the direction of the medium calorimeter producing peak incident heat fluxes of $\sim 200 \mathrm{~kW} / \mathrm{m}^{2}$.

Table 4. Medium Calorimeter Average Heat Flux.

\begin{tabular}{|c|c|c|c|c|}
\hline & Test 1 & Test 2 & Test 3 & Test 4 \\
\hline & $200-1500$ & $200-1500$ & $200-1500$ & $200-1500$ \\
\hline Duration (s) & $\begin{array}{c}\text { ave } \pm \mathrm{s} . \mathrm{d} . \\
\left(\mathrm{kW} / \mathrm{m}^{2}\right)\end{array}$ & $\begin{array}{c}\text { ave } \pm \mathrm{s} . \mathrm{d} . \\
\left(\mathrm{kW} / \mathrm{m}^{2}\right)\end{array}$ & $\begin{array}{c}\text { ave } \pm \mathrm{s} . \mathrm{d} . \\
\left(\mathrm{kW} / \mathrm{m}^{2}\right)\end{array}$ & $\begin{array}{c}\text { ave } \pm \mathrm{s} . \mathrm{d} . \\
\left(\mathrm{kW} / \mathrm{m}^{2}\right)\end{array}$ \\
\hline TC 1 (East) & $1 \pm 0$ & $63 \pm 65$ & $1 \pm 0$ & $33 \pm 53$ \\
\hline TC 2 & $1 \pm 0$ & $86 \pm 74$ & $1 \pm 0$ & $43 \pm 60$ \\
\hline TC 3 (Top) & $3 \pm 2$ & $111 \pm 65$ & - & - \\
\hline TC 4 & $6 \pm 3$ & $123 \pm 62$ & $5 \pm 1$ & $76 \pm 50$ \\
\hline TC 5 (West) & $5 \pm 3$ & $112 \pm 52$ & - & - \\
\hline TC 6 & $3 \pm 1$ & - & - & - \\
\hline TC 7 (Bottom) & $1 \pm 0$ & $67 \pm 44$ & $1 \pm 0$ & $35 \pm 28$ \\
\hline TC 8 & $1 \pm 0$ & $57 \pm 48$ & $1 \pm 0$ & $32 \pm 40$ \\
\hline
\end{tabular}

\section{Large Calorimeter Heat Fluxes}

Figure 6 presents polar plots of average incident heat flux at the four section measurement planes on the surface of the large calorimeter. In Test 1, the data is averaged over a duration of 300-1200 seconds, whereas in Test 2 the averaging is limited to the 200-800 seconds interval due to TC failures. For Test 1 , the incident heat fluxes to the large calorimeter were generally less than $80 \mathrm{~kW} / \mathrm{m}^{2}$, with interior sections 2 and 4 recording the highest flux. The largest heat fluxes were observed on the west side $\left(180^{\circ}\right)$ of the calorimeter, facing the pool. In Test 2, the incident heat fluxes to the large calorimeter were largest on the bottom and east sides of the calorimeter, averaging $140-160 \mathrm{~kW} / \mathrm{m}^{2}$. Video indicated vigorous fire plumes sweeping across and underneath the calorimeter. Peak heat fluxes of $\sim 200 \mathrm{~kW} / \mathrm{m}^{2}$ were measured. The large heat fluxes on the leeward side (away from the fire) of the large calorimeter were due to increase fuel/air mixing in the wake from interaction of wind with the calorimeter. 

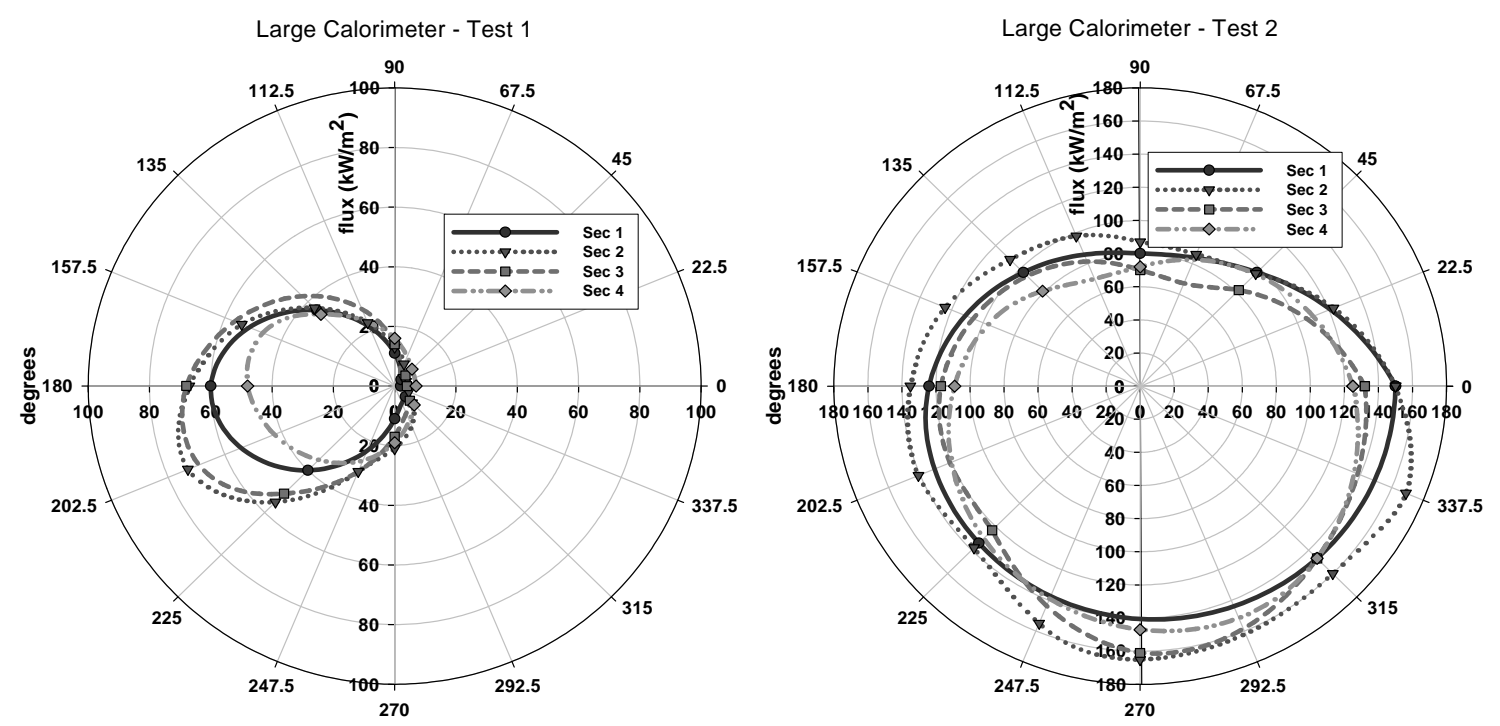

Fig. 6. Spatial distribution of incident heat flux to the large calorimeter.

\section{CONCLUDING REMARKS}

Four well-characterized open pool fire experiments were performed to provide data of sufficient quality for fire code validation purposes. The data sets have been fully documented [15] specifically with the end-user, the fire analyst, in mind. All of the experiment data is included on a DVD ROM [15]. Data and plots in Excel format provide a convenient interface for navigating the experiment data sets. In addition to the numerical and graphical presentation of the data, animations of heat flux contour plots of the fuel pool, terrain, and calorimeters were created to better visualize the fire insult. The DVD ROM also contains the digitized movies from the video camera coverage of all the fires. The data and the video media (available on request) are intended to provide a user-friendly archival database in support of model validation efforts.

The experiments measured the burning rate of liquid fuel in an open pool and the resultant heat flux to objects and the surrounding environment with well-characterized boundary and initial conditions. Experimental results showed a strong correlation between wind conditions, fuel mass loss rate, and incident heat flux to the pool and calorimeters. The incident heat flux to the pool was found to be significantly affected by the orientation of the fire plume with respect to the ground normal. For strong crosswinds, the highest heat fluxes were observed on the downwind side of the pool. Significantly smaller incident heat fluxes were observed on the windward side of the pool. When crosswinds were small, the largest incident heat fluxes occurred around $1 / 2$ the radius of the pool. Much lower heat fluxes were observed at or near the center of the pool, probably a result of an oxygen-starved interior.

\section{ACKNOWLEDGEMENT}

This study was sponsored by the Department of Defense - Defense Threat Reduction Agency and was performed at Sandia National Laboratories. This work was supported by the United States Department of Energy and was performed at Sandia National Laboratories. Sandia is a multiprogram laboratory operated by Sandia Corporation, a Lockheed Martin Company, for the United States Department of Energy under Contract No. DE-AC04-94AL85000.

\section{REFERENCES}

[1] Tieszen, S.R., Nicolette, V.F., Gritzo, L.A., Holen, J.K., Murray, D., and Moya, J.L., "Vortical Structures in Pool Fires: Observation, Speculation, and Simulation," SAND96-2607, Sandia National Laboratories, Albuquerque, NM., 1996. 
[2] Gritzo, L.A., Nicolette, V.F., Tieszen, S.R., and Moya, J.L., "Heat Transfer to the Fuel Surface in Large Pool Fires," Transport Phenomenon in Combustion, S.H. Chan, ed., Taylor and Francis, 1995.

[3] Nicolette, V.F., Gritzo, L.A., Moya, J.L., and Tieszen, S.R., “Comparison of Large JP4- and JP8Fueled Pool Fires," Proceedings of the International Conference on Fire Research and Engineering, Orlando, Florida, September 10-15, 1995, SFPE, Quincy, MA.

[4] Gritzo, L.A., Gill, W., and Nicolette, V.F., "Estimates of the Extent and Character of the OxygenStarved Interior in Large Pool Fires,” Very Large Scale Fires, ASTM STP 1336, N.R. Keltner, N.J. Alvares, and S.J. Grayson, Eds., American Society for Testing and Materials, 1998.

[5] Gritzo, L.A. and Moya, J.L., "Fire Characterization and Object Thermal Response for a Series of Tests with a Large Flat Plate Adjacent to Large JP4 Fuel Fire,” SAND97-0047, Sandia National Laboratories, Albuquerque, NM., 1997.

[6] Suo-Anttila, J.W. and Gritzo, L.A., "Thermal Measurements from a Series of Tests with a Large Cylindrical Calorimeter on the Leeward Edge of a JP-8 Pool Fire in Cross-Flow,” SAND20011986, Sandia National Laboratories, Albuquerque, NM., 2001.

[7] Gritzo, L.A., Nicolette, V.F., Moya, J.L., Skocypec, R.D., and Murray, D., "Wind-Induced Interaction of a Large Cylindrical Calorimeter and an Engulfing JP8 Pool Fire,” Proceedings of the Symposium on Thermal Sciences in Honor of Chancellor Chang-Lin Tien, Berkeley, CA, November 14, 1995.

[8] Blanchat, T.K., Humphries, L.L., and Gill, W., "Sandia Heat Flux Gauge Thermal Response and Uncertainty Models,” SAND2000-1111, Sandia National Laboratories, Albuquerque, NM, May 2000.

[9] Nakos, J.T., Suo-Anttila, J.M, and Gill, W., "Shroud boundary condition characterization experiments at the Radiant Heat Facility.”, SAND2004-5080, Sandia National Laboratories, Albuquerque, NM., 2004.

[10] Gill, W. and Nakos, J.T., "Temperature/Heat Flux Measurement Errors Caused by Thermocouple Shunt Impedance," accepted by the Ad Hoc Committee on Heat Flux Measurement and Calibration for presentation at the session "Case Studies in Heat Flux Measurements - Successes and Problems" at the 1999 International Mechanical Engineering Congress and Exposition (IMECE), Nashville, TN, November 1999.

[11] Coleman, H.W. and Steele, W.G., Experimentation and Uncertainty Analysis for Engineers, Wiley \& Sons, 1999.

[12] Drysdale, D., An Introduction to Fire Dynamics (2nd Edition), John Wiley \& Sons, New York, page 162, 1998.

[13] Nakos, J.T., "Uncertainty Analysis of Steady State Incident Heat Flux Measurements in Hydrocarbon Fuel Fires”, Sandia National Laboratories, SAND2005-7144, 2005.

[14] Brown, A.L. and Blanchat, T.K., “A Validation Quality Heat Flux Dataset for Large Pool Fires”, Proceedings of HT2003, ASME Summer Heat Transfer Conference, July 23, 2003, Las Vegas, NV.

[15] Blanchat, T.K., Nicolette, V.F., Sundberg, W.D., and Figueroa, V.G., "Well-Characterized Open Pool Experiment Data and Analysis for Model Validation and Development,” SAND2006-7508, Sandia National Laboratories, Albuquerque, NM, December 2006. 
